\title{
Loads on cutter teeth for removing asphalt pavement
}

\author{
Denis Furmanov", Vladimir Chizhov, Ivan Tyuremnov and Denis Troshin \\ Yaroslavl State Technical University, 88, Moskovskiy prospekt, Yaroslavl, 150023, Russia
}

\begin{abstract}
Machines and equipment for asphalt pavement milling are widely used throughout the world. With the growth of road construction, the demand for this class of machinery also rises. At the same time, the optimization of design and technological parameters of the milling machines is impossible without a comprehensive study of processes of milling asphalt concrete with the working elements of a cutter. The article provides a brief overview of different approaches to find out the loads on the working bodies of various machines, as well as indicates the advantages and disadvantages of these approaches. We describe the design of a pendulum stand used to study the processes of milling asphalt concrete with a single tooth cutter. We also describe the method of operation of such a stand. The design of the pendulum stand allows us to determine the cutting work, the average, and the instantaneous cutting forces. The work also provides the result of tests of cutting elements during their interaction with samples of asphalt concrete of different grades that are widely used in road construction. We also show the non-linear dependence of cutting resistance forces on the temperature and thickness of penetration of a cutting element. It is noted that working with a slight tooth penetration leads to low energy efficiency of the work process. It is also noted that temperature affects cutting resistance forces significantly less in asphalt concrete containing high amount of gravel.
\end{abstract}

\section{Introduction}

Rotary hoes and equipment for asphalt concrete regeneration are widely used around the world. While the operational durability of asphalt concrete increases, the more powerful machinery and equipment is produced, the milling speed and performance also increase.

Rotary hoes is a large class of equipment with different performance, functionality, drive type, and underlying hardware. The design of such machines should be based on reliable data on the working process of interaction of a cutting tooth with the material, as well as the correct mathematical description of the cutter's operation, given the complex set of trajectories of a cutting tooth, geometric parameters of a drum, the impact of the amount of cutter teeth and their placement on the drum. The second part of the equipment design task is studied well enough at this moment. A large number of works dedicated to

\footnotetext{
* Corresponding author: denis_furmanov@mail.ru
} 
calculation of geometric, force and energy parameters of milling equipment allows us to calculate the cutters for various materials with known values of cutting forces $[1,2,3]$

The issue of determining the forces themselves is studied not that well. The cutting forces value is affected by a large number of parameters: geometric shape and position of the cutting element; the chip thickness of the material; the presence, durability, and the amount of stone fractions; the temperature of asphalt concrete. All this determines the cutting force as a complex function, the arguments of which are all the parameters mentioned above. The errors that occur due to wrong prediction of cutting forces will definitely lead to loss of productivity, incorrect choice of the power unit, incorrect justification of the strength of equipment elements and, possibly, failure of the entire machine. Let us try to understand this issue based on previous studies.

According to the classification presented in $[4,5]$, asphalt concrete is a complex dispersed and filled composite material with a matrix made from a mixture of bitumen and modifiers, and, in some cases, with sand, while filled with stone fraction. Structure formation of asphalt concrete begins in a mixing chamber of an asphalt mixer and continues in the long operation process under the loads of wheeled vehicles and environmental factors $[6,7]$. All of this constantly changes durability of asphalt concrete and affects the cutting resistance.

There are several groups of works dedicated to the issue of determining forces of cutting resistance of road construction materials, which can arbitrarily be divided into three groups.

The core of the first group consists of the works based on analytical study of the process by analyzing rheological models [8], on the analysis of limit equilibrium of an adhesive granular medium theory [9], as well as using parts of the similarity law [10].

The use of these methods, relating to determining asphalt cutting resistance forces, has not found wide application due to complexity of mathematical models and the need to determine other values, such as adhesion, modulus of elasticity, viscosity, the method of determination and forecast accuracy of which is difficult to judge. Given that any object modeling, including mathematical modeling, supposes the existence of an error in the calculation results, the use of values as parts of these modules determined with limited precision will eventually lead to an unacceptable error in the final results.

Therefore, the practical value is in the works based on experimental studies. The works of A. N. Zelenin [11] show the estimation of resistance forces of the material to cutting with simple punches for soils of different durability. Based on these studies, the empirical equations to determine the forces of cutting resistance of this group of materials are created. To determine the cutting resistance of solid rocks, the work [12] employs experimental methods. These works were developed in the form of real machinery and equipment and formed the basis for further works with great practical importance [13, 14].

In relation to the study of the process of destruction of asphalt concrete, there are some studies with high practical significance formed by works based on experimental determination of parameters of a working process. In particular, the works [15. 16] provide the results of determining the specific values to determine the forces of cutting resistance of asphalt concrete by cutting elements of disc cutters. It is difficult to overestimate these works of V. F. Kulepov. They are of great practical importance and can be a good basis for the development of milling equipment. The drawback of these works lies in new types of asphalt concrete and significantly higher material cutting speeds of modern machinery. The design of cutting elements has also changed.

[17] provides the calculated dependencies that allow us to determine the loads on a cutting element. However, the aforementioned data allow us to estimate the impact of speed on cutting force at cutting speeds up to $0.9 \mathrm{~m} / \mathrm{s}$; however, the existing milling machines have long been working at cutting speeds up to $3 \ldots 4 \mathrm{~m} / \mathrm{s}$. Additionally, it is unclear under 
what conditions (what types of asphalt concrete and cutting elements) these data were obtained.

\section{Problem statement}

The drawbacks of the works mentioned require the specification of the basic parameters of a working process of asphalt milling, which, in turn, requires additional experimental studies.

Making an adequate estimation of asphalt concrete milling process is possible only if the asphalt concrete cutting process with a single cutting tooth is studied in detail. Thus, it is important to draw up and experiment with real samples of asphalt concrete that is widely used in road construction, all the while using real cutting elements at cutting speeds and chip thickness comparable to the parameters of milling machines widely used in road construction.

\section{Study methods}

The most complete experiment with the aforementioned restrictions is possible with a pendulum stand (Fig. 1). The stand is a built-up structure consisting of a pendulum 1, on which a cutting element 2 is mounted via a hinged platform 5 . The material sample 3 is fixed to a separate rigid frame. The forces appearing during the cutting process are transferred through the platform 5 to the level 6 and, consequently, are received by a force sensor 7 through the gear gate.

The pendulum is deflected through the elevation angle 1 and is dropped with a special coupling device. The cutting element does the work of cutting forces at the bottom point, while the energy remaining after the impact allows the pendulum to keep moving deflecting through the deviation angle 2. Naturally, the deflection angle will correspond to a specific value of residual energy after the impact. According to the energy conservation law and ignoring friction forces at the support of the pendulum and other minor resistances, we can formulate the following dependency:

where $\mathrm{h} 1$ is the lifting height, $\mathrm{m}$;

$$
A=m \cdot g \cdot\left(h_{1}-h_{2}\right)
$$

$\mathrm{h} 2$ is the deflection height after impact, $\mathrm{m}$;

$\mathrm{m}$ is the reduced pendulum mass, $\mathrm{kg}$;

$\mathrm{g}=9.81 \mathrm{~m} / \mathrm{s} 2$ is the gravity acceleration.

The heights $\mathrm{h} 1$ и $\mathrm{h} 2$ are determined with the elevation and deviation angles 1 and 2 . Based on these expressions, we can convert the equation (1) to:

where $\mathrm{L}$ is the pendulum length, $\mathrm{m}$.

$$
A=m \cdot g \cdot L \cdot\left(\cos \alpha_{2}-\cos \alpha_{1}\right)
$$

We need to determine the given mass of the pendulum $\mathrm{m}$ to move from a physical to a mathematical pendulum, assuming that the mass is concentrated at the cutting point. For this purpose, the following dependency is used

where $\mathrm{T}$ is the pendulum period, $\mathrm{s}$;

$$
\mathrm{m}=\mathrm{T}^{2} \cdot \mathrm{M} /\left(4 \cdot \pi^{2} \cdot \mathrm{L}^{2}\right)
$$

$\mathrm{M}$ is the first moment of the pendulum, $\mathrm{kg} \cdot \mathrm{m}$;

The parameters $\mathrm{T}$ and $\mathrm{M}$ are measurable in this stand.

After determining the work of cutting forces A we can determine the values of their average cutting force $\mathrm{F}_{0}$ :

$$
\mathrm{F}_{0}=\mathrm{A} / \mathrm{b}
$$


By determining the work of cutting resistance forces and average cutting forces, we can prove the energy parameters of the process, determine the drive power required for the given rotor design. However, the details of the cutting drum and the cutting elements during the work inevitably suffer the shock loads. Wrong assessment of dynamic effects will inevitably lead to a loss of durability, and considering that the vast majority of rotors is a welded structure, the impact of fatigue destruction on welded joints can lead to a costly failure of the rotor in a small timeframe.

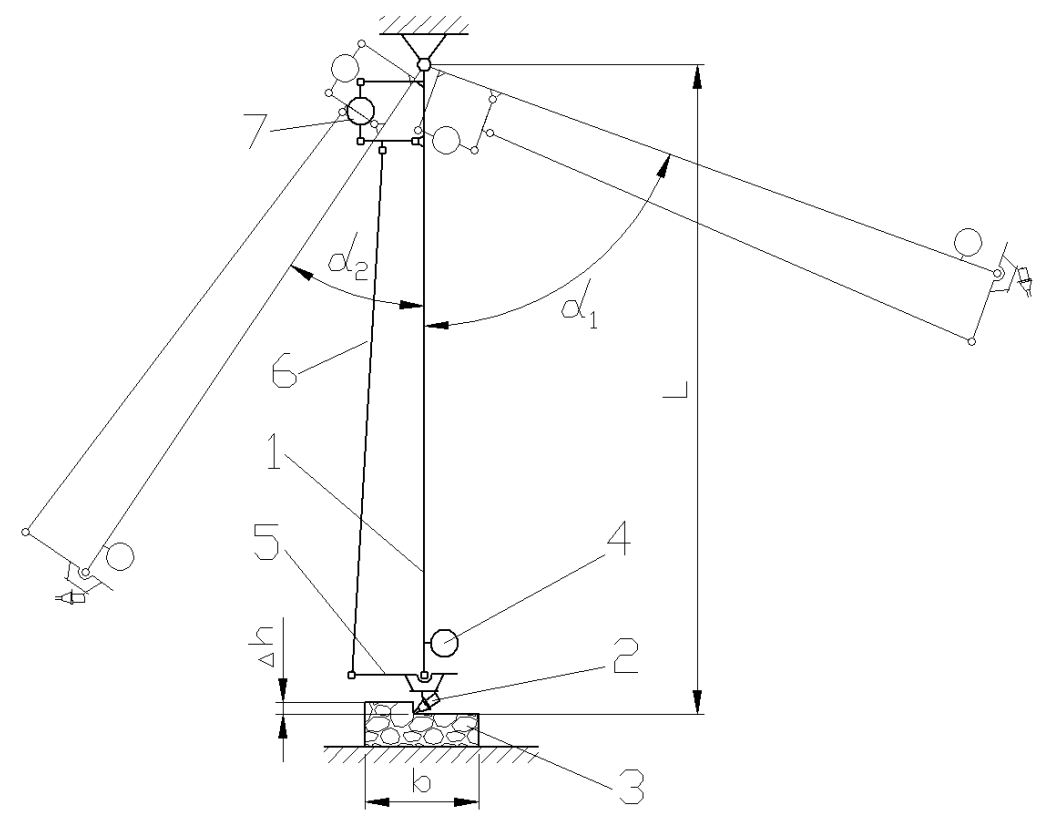

Fig. 1. A pendulum to study the working process of asphalt concrete destruction by working elements of milling machines.

To determine the instantaneous cutting resistance forces, the pendulum is additionally equipped with a force sensor installed at the top of the pendulum. The value of cutting resistance forces is recorded to a computer via an analog-to-digital converter (Fig. 2).

We chose granular asphalts for testing, which are widely used in construction. The asphalt grades SMA-20 [18], B-2, B-3, and MZP-2 [19] were used. The aforementioned asphalts contain crushed granite graded M1200 [20] and bitumen BND 60/90 [21] with the additives of dolomite powder or the modifiers.

The tests were conducted at initial cutting speeds of $4 \mathrm{~m} / \mathrm{s}$ with variable chip thickness. The asphalt temperature was changed by using the temperature control system in a refrigerated chamber and in an oven for two hours. The method of planning [22] was used for each set of samples. We chose symmetrical two-factor experiment plans and obtained the response value in the extreme and middle points.

\section{Results}

The results of the tests are shown in the following graphs (Fig. 3). Statistical processing of the results was performed by fitting the coefficients to the following regression equation:

$$
\mathrm{F}=\mathrm{a}_{0} \cdot \Delta \mathrm{h}^{\mathrm{a} 1} \cdot \mathrm{t}^{\mathrm{a} 2}
$$

where $\mathrm{F}$ is the cutting resistance force, $\mathrm{N}$; 
$\Delta \mathrm{h}$ is the chip thickness, $\mathrm{mm}$;

$\mathrm{t}$ is the asphalt temperature, ${ }^{\circ} \mathrm{C}$;

$\mathrm{a}_{0}, \mathrm{a}_{1}, \mathrm{a}_{2}$ are the beta coefficients.

The regression equations received are presented in Table 1.

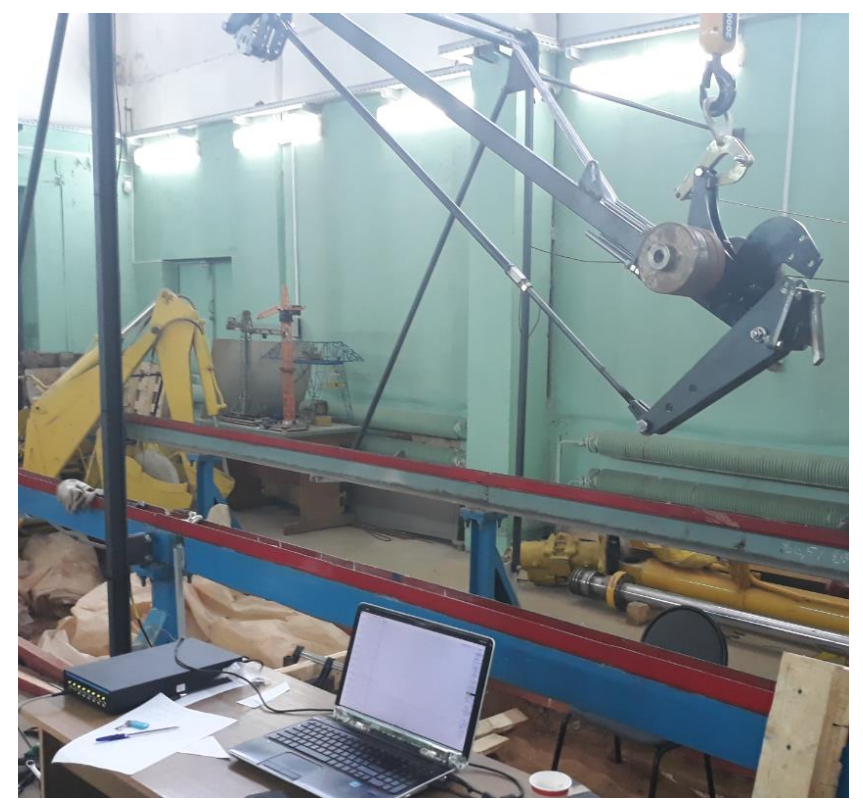

Fig. 2. Pendulum stand

Table 1. The regression dependencies of cutting forces on the chip thickness and temperature for different grades of asphalt concrete

\begin{tabular}{|c|c|c|}
\hline Asphalt concrete grade & Regression equation & Confidence factor $\mathbf{R}^{\mathbf{2}}$ \\
\hline B2 & $\mathrm{F}=1971 \cdot \Delta \mathrm{h}^{0.45} \cdot \mathrm{t}^{-0.445}$ & 0.804 \\
\hline V3 & $\mathrm{F}=43382 \cdot \Delta \mathrm{h}^{0.672} \cdot \mathrm{t}^{-1.755}$ & 0.865 \\
\hline MZP-2 & $\mathrm{F}=4438 \cdot \Delta \mathrm{h}^{0.224} \cdot \mathrm{t}^{-0.430}$ & 0.810 \\
\hline SMA-20 & $\mathrm{F}=900.4 \cdot \Delta \mathrm{h}^{0.45} \cdot \mathrm{t}^{-0.75}$ & 0.758 \\
\hline
\end{tabular}

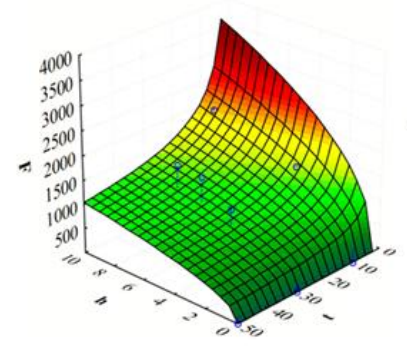

a

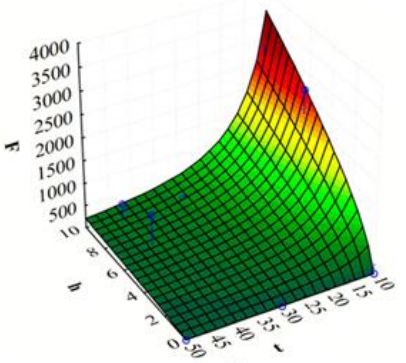

b

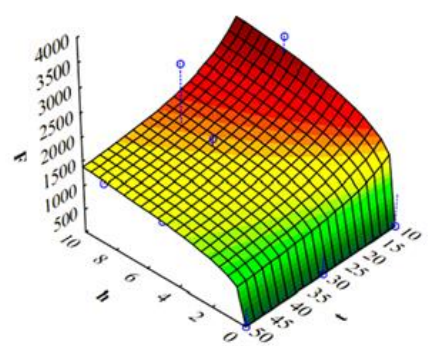

c

$\mathrm{a}$ - the B2 grade asphalt, b - the V3 grade asphalt, c - the MZP-2 grade asphalt.

Fig. 3. The results of the studies of cutting resistance forces for different grades of asphalt depending on the chip thickness and temperature. 
High degree of the confidence factors provides a satisfactory correlation and the possible use of the results in the mathematical modeling of the working process of milling asphalt.

\section{Conclusions}

Based on the experimental work performed, we can reasonably state the following:

1. The function of the cutting resistance force, depending on the chip thickness, is nonlinear.

2. With a relatively small chip thickness, the power consumption of the process is quite high, considering the sharp increase of the cutting resistance forces. The greatest efficiency of the cutting tools is achieved when the chip thickness is above three millimeters.

3. The temperature of asphalt concrete has a large impact on the cutting resistance, especially for asphalts with high content of bitumen.

4. The asphalts with high content of gravel, the temperature has a significantly smaller impact; however, the values of cutting resistance forces are quite high themselves. This fact makes the process of hot milling ineffective.

5. The energy consumption of the asphalt cutting process greatly depends on the amount of stone fraction in it and the temperature.

The obtained dependencies allow us to draw up a mathematical model of the process of asphalt cutting with milling working bodies of road construction machines, to justify, and to optimize their parameters.

\section{References}

1. Yu. M. Gavrilov, I. A. Osmin, Excavating and milling machines: (Yaroslavl, 1985)

2. L. N. Samsonov, Milling peat deposits (Moscow, Nedra, 1985)

3. A. Karoshkin, A. V. Krasnoludskiy, Determination of power consumption of milling process with a cutter with complex tooth movement (Tula State University, Tula, 2003, $\mathrm{p}$ 167)

4. S.L. Bazhenov, Mechanics and technology of composite materials: Scientific edition. (Dolgoprudny, Publishing House 'Intellect', 2014)

5. F. L. Matthew, R. D. Rawlings. Composite materials. Engineering and Science. (Moscow, Technosphera. 2004)

6. I. A. Rybyev, Construction materials based on binders: artificial construction conglomerates (Moscow, Vysshaya Shkola. 1978)

7. L.B. Gezenzvey, Road asphalt (Moscow, Transport publ. house, 1976)

8. Ya. Aizenshtok. On the physical theory of soil cutting (mining journal, 1949, No. $5 \mathrm{p}$ 16)

9. V. V. Sokolovsky, Statics of granular medium (Moscow, Gostehteorizdat, 1954)

10. V. I. Balovnev, Physical modeling of soil cutting (Moscow, Mashinostroenie publ. house, 1969)

11. A. N. Zelenin, Basics of soil destruction by mechanical means (Moscow, Mashinostroenie publ. house, 1968)

12. Ju. A. Vetrov, V. L. Baladinskiy, V. F. Barannikov, V. P. Kuksa, Solid soil destruction (Kyiv, Budivelnyk, 1972)

13. P. Berkman, A. V. Rannev, A. K. Reish, One-bucket hydraulic excavators (Moscow, Vysshaya Shkola, 1973) 
14. E. V. Kurilov, D. I. Troshin Dozer productivity improvement: the experience in design and operation of hemispherical dozer blades with movable side walls (Yaroslavl. Publishing House YSTU, 2018)

15. V. N. Bibikov. N. N. Tarbaev, V. F. Kulepov, The impact of mechanical properties of asphalt concrete on the wear of the cutting tool during milling. (Tr. Gorky Polytechnic Institute. Gorky, 1975, Vol. 31, v. 8, p 11)

16. V. F. Kulepov, Experimental study of loads on disc cutting working member during the opening of asphalt concrete pavements (Gorky, Gorky regional schientific and technical conference, 1983, p 27)

17. M. P. Baratashvili. Determination of factors influencing the operation modes of a machine and their importance for destruction of the surface asphalt layers (Scientific digital archive. URL: http://econf.rae.ru/article/6606, access date: 11.02.2019).

18. GOST 31015-2002, Bituminous stone mastic mixtures and stone mastic asphalt. Specifications. (Introduced from 1.05.2003 Moscow, Standartinform, 2003)

19. GOST 9128-2009, Asphaltic concrete mixtures for roads and aerodromes and asphaltic concrete. Specifications. (Introduced from 1.01.2009, Moscow, Standartinform, 2010)

20. GOST 8267-93, Crushed stone and gravel of solid rocks for construction works. Specifications (Introduced from 10.11.1993 Moscow, Standartinform, 1994)

21. GOST 22245-90, Viscous petroleum road bitumens. Specifications. (Moscow.: IPC Publishing House of Standards, 2005)

22. G. Zedgenidze. Experiment planning for the research of multicomponent systems (Moscow, Nauka, 1976) 